\title{
Neonatal Vaginal Discharge
}

National Cancer Institute

\section{Source}

National Cancer Institute. Neonatal Vaginal Discharge. NCI Thesaurus. Code $C 117314$.

Secretions which may be thin or tenacious, mucoid or glairy, grayish or milky, occasionally blood tinged, that originate in the vagina and are a consequence of the residual influence of maternal estrogen. 